\title{
Surface Waves Excitation in Magnetized Spin Quantum Plasmas
}

\author{
Bahaa F. Mohamed ${ }^{*}$, Salah Y. Elbakry², Abrar A. Salah ${ }^{2}$ \\ ${ }^{1}$ Plasma Physics Department, N.R.C., Atomic Energy Authority, Cairo, Egypt \\ ${ }^{2}$ Department of Physics, Faculty of Science, Ain-Shams University, Cairo, Egypt \\ Email: *mohamedbahf@yahoo.co.uk
}

Received 25 December 2015; accepted 23 February 2016; published 26 February 2016

Copyright (C) 2016 by authors and Scientific Research Publishing Inc.

This work is licensed under the Creative Commons Attribution International License (CC BY).

http://creativecommons.org/licenses/by/4.0/

(c) (i) Open Access

\begin{abstract}
The influence of the intrinsic spin of electrons on the excitation of transverse electromagnetic surface waves in magnetized plasma is considered. We use a fluid formalism to include quantum corrections due to the Bohm potential and magnetization energy of electrons due to its spin. The effects of both quantum corrections are shown in the dispersion relation for the propagation of surface waves. Also, it is found that the phase and group velocities are increased due to the quantum effects. In the nonrelativistic motion of electrons, the spin effects become noticeable even when the external magnetic field is relatively low.
\end{abstract}

\section{Keywords}

\section{Quantum Plasma, Spin Electron, Quantum Effects}

\section{Introduction}

Already in the 1960's, the excitation spectrum of quantum plasmas was studied by Pines. Since Pines's pioneering paper, a number of researchers studied the theoretical quantum statistical properties of plasmas [1]. Dense plasmas composed of degenerate electrons, ions, positrons, and/or holes are referred to as quantum plasmas. In the latter, the degeneracy of the lighter plasma species (electrons, positrons, holes) appears at relatively low temperatures and very high densities, where the mean inter-particle distance is of the same order of the de Broglie thermal wavelength [2]. Recently, so called quantum plasmas have received attention, in which the plasma particles' quantum properties are taken into account [3]-[8]. The field of quantum plasma is becoming of increasing current interest, motivated by its applications in modern technology [9]-[11]. Different research in quantum plasma has been presented such as quantum plasma, magnetoplasma, dusty plasma and laser plasma

\footnotetext{
*Corresponding author.
} 
[12] [13]. The studies have been motivated by the recent development in quantum wells and nanostructured materials [14], the discovery of ultracold plasmas, astrophysical applications [15], or a general theoretical interest [16]-[20]. There are various models to study quantum effects in plasma, for example the popular QHD model and the Wigner-Poisson system. The QHD model is a generalization of the fluid model for plasmas with the inclusion of quantum correction term known as Bohm potential. This term is responsible for the electron tunneling at nano-scale as well as for introducing new types of plasma waves in dense quantum plasmas [21]. Within fluid theory, the theoretical models applied cover effects such as the Bohm potential, and the Fermi pressure. The magnetization current associated with the electron spin and the magnetic dipole force have also been captured within fluid models, with a macroscopic spin density [22]. For wavelengths much longer than the characteristic de Broglie length of the particle, the quantum effects associated with particle dispersive effects disappear, and the evaluation equation is much simplified. The remaining quantum effects are all due to the electron spin, explicitly due to terms being proportional to the magnetic moment in the dynamic equations, or indirectly due to the background distribution function obeying Fermi-Dirac statistic [22]. The spin properties of electrons can be important even in high temperature plasmas of modest magnetic field strength and density [23]. The spin properties of the material constituents also make its presence felt through collective effects [24].

Several authors incorporated the effect of the dispersion caused by the quantum Bohm potential and/or the spin effect in the study of plasma waves [12] [25] [26]. Misra A. P., et al [25] investigated the nonlinear propagation of electromagnetic electron-cyclotron waves along an external magnetic field, and their modulation by electrostatic small but finite amplitude ion-acoustic density perturbations in uniform quantum plasma with intrinsic spin of electrons. The effects of the quantum force associated with the Bohm Potential and the combined effects of the classical as well as the spin-induced ponderomotive force are taken into account. Asenjo, F. A. [26] studied the quantum corrections to the oblique propagation of the magnetosonic waves in a warm magnetoplasma composed by mobile ions and electrons. Shukla P. K. [12] derived the linear dispersion relation for elliptically polarized electromagnetic waves in a dense fermionic quantum magnetoplasma, taking into account the quantum forces associated with the quantum Bohm potential and the magnetization energy of the electrons due to the $1 / 2$-electron spin effect.

In the present work, we study the quantum effects introduced by both the Bohm potential and the spin of electrons in propagation of surface waves in electron magnetoplasma. We will derive and analyze the dispersion relation of the excitation of transverse electromagnetic (TM) surface waves by using fluid (QHD) model.

\section{Model of Equations}

We consider a homogeneous quantum plasma half-space under an external strong magnetic field $\boldsymbol{B}_{0}=B_{0} \boldsymbol{e}_{z}$. In order to study the TM surface waves in dense Fermi-Dirac plasma, we assume that the quantum statistical pressure, the quantum tunneling effect and the magnetization energy of the electrons due to the 1/2-electron spin effect are included while immobile ions form the neutralizing background.

By employing the QHD model, we can write the dynamic equations of an electron as follows:

$$
m n\left[\frac{\partial \boldsymbol{v}}{\partial t}+(\boldsymbol{v} \cdot \nabla) \boldsymbol{v}\right]=q n\left[\boldsymbol{E}+\boldsymbol{v} \times\left(\boldsymbol{B}+\boldsymbol{B}_{0}\right)\right]-\nabla P+n \boldsymbol{F}_{Q}
$$

where,

$$
\boldsymbol{F}_{Q}=\frac{\hbar^{2}}{2 m} \nabla\left(\frac{\nabla^{2} \sqrt{n}}{\sqrt{n}}\right)-\eta(\alpha) \mu_{B} \nabla B
$$

$\boldsymbol{F}_{Q}$ is the quantum force due to the different quantum effects. The first term in the Equation (2) representing the Bohm potential and the second term due to the dynamics of spin electrons. $\boldsymbol{E}, \boldsymbol{B}$ and $\boldsymbol{B}_{0}$ are the induced electric, magnetic fields and constant external strong magnetic field in the z-direction, respectively. Also, equation of continuity is:

$$
\frac{\partial n}{\partial t}+\operatorname{div}(n v)=0
$$

Here $n, \boldsymbol{v}$ and $m$ are the number density, the velocity and the mass of electron respectively and $h$ is the Plank's constant divided by $2 \pi$. The electrons obey the following pressure law which represents the equation 
of state in Fermi gas:

$$
P=2 n T_{F e}, \eta(\alpha)=2 \tanh (\alpha), \alpha=\frac{\mu_{B} B_{0}}{T_{F e}} \text { and } \mu_{B}=\frac{e \hbar}{2 m c}
$$

We now study the response of the system to any small perturbations and assume that every physical quantity $\psi$ representing $(n, v, E, B)$ has this form $\psi=\psi_{0}+\psi_{1}$, where $\psi_{0}$ is the unperturbed value and $\psi_{1}$ is a small perturbation, with $\left(\psi_{1} \ll \psi_{0}\right)$ with varying like $\psi(x) \exp (i \boldsymbol{K} \cdot \boldsymbol{r}-i \omega t)$ with $\boldsymbol{K}_{k_{y}} \boldsymbol{e}_{y}$.

The electromagnetic fields of TM surface waves $E_{x}, E_{y}$ and $B_{z}$ are given by the Maxwell-Poisson equations.

$$
\begin{aligned}
& \nabla \cdot \boldsymbol{E}=-4 \pi e n \\
& \nabla \cdot \boldsymbol{B}=0 \\
& \nabla \wedge \boldsymbol{E}=-\frac{1}{c} \frac{\partial \boldsymbol{B}}{\partial t} \\
& \nabla \wedge \boldsymbol{B}=\frac{1}{c} \frac{\partial \boldsymbol{E}}{\partial t}+\frac{4 \pi}{c} \boldsymbol{J}
\end{aligned}
$$

The Equation (1) can be linearized, by assuming that the equilibrium quantities $\boldsymbol{E}_{o}=\boldsymbol{B}_{o}=\boldsymbol{v}_{o}=0$, to obtain the velocity components of the plasma electrons as follows:

$$
\begin{aligned}
v_{x}= & \frac{-i \omega}{m\left(\omega^{2}-\omega_{c}^{2}\right)}\left[e E_{x}+\frac{m v_{F e}^{2} Q}{n_{0}}\left(\frac{\partial n}{\partial x}\right)+\eta(\alpha) \mu_{B} \frac{\partial B_{z}}{\partial x}\right] \\
& -\frac{\omega_{c}}{m\left(\omega^{2}-\omega_{c}^{2}\right)}\left[e E_{y}+\frac{i k_{y} m v_{F e}^{2} Q}{n_{0}} n+i k_{y} \eta(\alpha) \mu_{B} B_{z}\right] \\
v_{y}= & \frac{-i \omega}{m\left(\omega^{2}-\omega_{c}^{2}\right)}\left[e E_{y}+\frac{i m k_{y} v_{F e}^{2} Q}{n_{0}} n+i k_{y} \eta(\alpha) \mu_{B} B_{z}\right] \\
& +\frac{\omega_{c}}{m\left(\omega^{2}-\omega_{c}^{2}\right)}\left[e E_{x}+\frac{m v_{F e}^{2} Q}{n_{0}} \frac{\partial n}{\partial x}+\eta(\alpha) \mu_{B} \frac{\partial B_{z}}{\partial x}\right] \\
v_{z}= & 0
\end{aligned}
$$

The Equations (8)-(10) display that Bohm potential and the spin quantum effects enhance the perturbation velocity of the electrons. The continuity Equation (3) yields the following wave equation for the perturbed electron density:

$$
\begin{aligned}
& \frac{\partial^{2} n}{\partial x^{2}}-k_{y}^{2}\left[1-\frac{\left(\omega^{2}-\omega_{c}^{2}\right)}{k_{y}^{2} v_{F e}^{2} Q}\right] n \\
& =\frac{-e n_{0}}{m v_{F e}^{2} Q}\left[\frac{\partial E_{x}}{\partial x}-\frac{i \omega_{c}}{\omega} \frac{\partial E_{y}}{\partial x}-\frac{k_{y} \omega_{c}}{\omega} E_{x}+i k_{y} E_{y}\right]-\frac{n_{0} \eta(\alpha) \mu_{B}}{m v_{F e}^{2} Q}\left[\frac{\partial^{2} B_{z}}{\partial x^{2}}-k_{y}^{2} B_{z}\right]
\end{aligned}
$$

where, $\omega_{p}^{2}=4 \pi e^{2} n_{o} / m$ and $\omega_{c}=e B_{o} / m$ are the electron plasma frequency and Larmor frequency. Also, $Q=1+\frac{\hbar^{2} k_{y}^{2}}{4 m^{2} v_{F e}^{2}}$.

To obtain the above Equations (7)-(11), the very slow nonlocal variation are neglected, (i.e., $\left.\partial^{4} / \partial x^{4} \ll \partial^{2} / \partial x^{2} \ll k_{y}^{2}\right)$. Also, from Maxwell's equations, the electric field components and the wave equation of the magnetic field component for these TM surface modes are obtained in the following forms:

$$
E_{x}=\chi_{1} \frac{\partial B_{z}}{\partial x}-k_{y} \varepsilon_{2} \chi_{2} B_{z}+\varepsilon_{1} \chi_{3} \frac{\partial n}{\partial x}+\varepsilon_{2} \chi_{3}\left(\frac{\omega_{c} k_{y}}{\omega}\right) n
$$




$$
\begin{aligned}
& E_{y}=-i \varepsilon_{2} \chi_{2} \frac{\partial B_{z}}{\partial x}+i k_{y} \chi_{1} B_{z}+i \varepsilon_{2} \chi_{3}\left(\frac{\omega_{c}}{\omega}\right) \frac{\partial n}{\partial x}+i k_{y} \varepsilon_{1} \chi_{3} n \\
& \frac{\partial^{2} B_{z}}{\partial x^{2}}-\aleph_{1}^{2} B_{z}=\left(\frac{\omega_{c}}{\omega}\right)\left(\frac{\chi_{3}}{\chi_{2}}\right)\left(\frac{\partial^{2} n}{\partial x^{2}}-k_{y}^{2} n\right)
\end{aligned}
$$

where, $\aleph_{1}^{2}=k_{y}^{2}-\frac{\omega}{c \varepsilon_{2} \chi_{2}}, \Delta=1-\frac{\omega_{p}^{4} \omega_{c}^{2}}{\omega^{2}\left(\omega^{2}-\omega_{h}^{2}\right)^{2}}, \quad \omega_{h}^{2}=\omega_{p}^{2}+\omega_{c}^{2}$,

$$
\begin{aligned}
& \varepsilon_{1}=1+\frac{\omega_{p}^{2} \omega_{c}^{2}}{\omega^{2}\left(\omega^{2}-\omega_{h}^{2}\right)}, \varepsilon_{2}=\frac{\left(\omega^{2}-\omega_{c}^{2}\right)}{\left(\omega^{2}-\omega_{h}^{2}\right)}, \\
& \chi_{1}=\frac{c \omega_{p}^{2}}{\omega \Delta\left(\omega^{2}-\omega_{h}^{2}\right)}\left[\frac{\hbar \omega \varepsilon_{1}}{m c^{2}}(\tanh \alpha)-\frac{\omega_{c} \varepsilon_{2}}{\omega}\right], \\
& \chi_{2}=\frac{c}{\omega \Delta}\left[1-\frac{\hbar \omega_{c} \omega_{p}^{2}}{m c^{2}\left(\omega^{2}-\omega_{h}^{2}\right)}(\tanh \alpha)\right], \chi_{3}=\frac{4 \pi e v_{F e}^{2} Q}{\Delta\left(\omega^{2}-\omega_{h}^{2}\right)} .
\end{aligned}
$$

By substituting the values of $E_{x}$ (Equation (12)) and $E_{y}$ (Equation (13)) in Equation (11), we can get on the wave equation of the perturbed density in the following form:

$$
\frac{\partial^{2} n}{\partial x^{2}}-\aleph_{2}^{2} n=S\left(\frac{\partial^{2} B_{z}}{\partial x^{2}}-k_{y}^{2} B_{z}\right)
$$

where, $\aleph_{2}^{2}=k_{y}^{2}-\frac{\left(\omega^{2}-\omega_{c}^{2}\right)}{v_{F e}^{2} Q L}, \quad S=\frac{e n_{0} c}{m \omega v_{F e}^{2} Q}\left[\frac{\omega_{c} \varepsilon_{2}^{2}}{\Delta L \omega}-\frac{\hbar \omega}{m c^{2}}(\tanh \alpha)\right]$,

$$
L=1+\frac{\omega_{p}^{2}}{\Delta\left(\omega^{2}-\omega_{h}^{2}\right)}\left[1+\frac{\omega_{c}^{2}\left(\omega^{2}+\omega_{p}^{2}-\omega_{c}^{2}\right)}{\omega^{2}\left(\omega^{2}-\omega_{h}^{2}\right)}\right] .
$$

It is worth to notice that the parameters $\chi_{1}, \chi_{2}$ and $S$ including the spin effect which strongly acts on the density and the electric and magnetic fields of the excited surface modes. Also, the excitation of these surface waves in our case are related to the following two conditions:

$$
\frac{\omega^{2} \Delta}{k_{y}^{2}}\left(\frac{\omega^{2}-\omega_{h}^{2}}{\omega^{2}-\omega_{c}^{2}}\right)+\frac{\hbar \omega_{c} \omega_{p}^{2}}{m\left(\omega^{2}-\omega_{h}^{2}\right)}(\tanh \alpha) \leq c^{2} \text { and } \omega^{2} \leq \omega_{c}^{2}+k_{y}^{2} v_{F e}^{2} Q L
$$

The inhomogeneous differential Equations (13) and (14) are dependent relations. We have solved them together (as in Ref. [27]) to obtain:

$$
\begin{aligned}
& n=A_{1}\left[1-\left(\frac{S}{L}\right)\left(\frac{\omega_{c}}{\omega}\right)\left(\frac{\chi_{3}}{\chi_{2}}\right)\left(\frac{\aleph_{1}^{2}-k_{y}^{2}}{\aleph_{1}^{2}-\aleph_{2}^{2}}\right)\right] \exp \left(-\aleph_{1} x\right)-A_{2}\left(\frac{S}{L}\right)\left(\frac{\aleph_{2}^{2}-k_{y}^{2}}{\aleph_{1}^{2}}\right) \exp \left(-\aleph_{2} x\right) \\
& B_{z}=A_{2} \exp \left(-\aleph_{2} x\right)+A_{1}\left(\frac{\omega_{c}}{\omega}\right)\left(\frac{\chi_{3}}{\chi_{2}}\right)\left(\frac{\aleph_{1}^{2}-k_{y}^{2}}{\aleph_{1}^{2}-\aleph_{2}^{2}}\right) \exp \left(-\aleph_{1} x\right), x>0 \\
& B_{z}=A_{3} \exp \left(\aleph_{v} x\right), x<0
\end{aligned}
$$

where, $\aleph_{v}^{2}=k_{y}^{2}\left(1-\frac{\omega^{2}}{c^{2} k_{y}^{2}}\right)$.

Here, $A_{1}$ and $A_{2}$ are the constants which describe the amplitudes of the perturbed field and density inside the plasma and $A_{3}$ is the amplitude of the excited magnetic field in the vacuum. From the boundary conditions at $x=0$, which consist in the vanishing of the fields and the normal component of the electron velocity on the 
boundary, we can get the amplitudes ( $A_{1}$ and $A_{2}$ ) inside the plasma region from the following relations:

$$
\begin{aligned}
& A_{1}=\left(\frac{\omega}{\omega_{c}}\right)\left(\frac{\chi_{2}}{\chi_{3}}\right)\left(\frac{\aleph_{1}^{2}-\aleph_{2}^{2}}{\aleph_{1}^{2}-k_{y}^{2}}\right)\left[\frac{\left(\aleph_{2}+\aleph_{v}\right)}{\left(\aleph_{2}-\aleph_{1}\right)}\right] A_{3} \\
& A_{2}=\frac{A_{3}}{\left[1-\left(\frac{\aleph_{2}+\aleph_{v}}{\aleph_{1}+\aleph_{v}}\right)\right]}
\end{aligned}
$$

The general dispersion relation for the electromagnetic surface waves in quantum magnetized plasma has also been obtained as follows:

$$
\begin{aligned}
& Z_{1}\left\{k_{y}\left[\left(\varepsilon_{1}+\varepsilon_{2}\right)\left(\frac{\omega_{c}}{\omega}\right)\right]-\aleph_{1}\left[\varepsilon_{1}+\varepsilon_{2}\left(\frac{\omega_{c}}{\omega}\right)^{2}\right]+\frac{\Delta\left(\omega^{2}-\omega_{h}^{2}\right)}{\omega_{p}^{2}}\left[k_{y}\left(\frac{\omega_{c}}{\omega}\right)-\aleph_{1}\right]\right\} \\
& -Z_{2}\left\{k_{y}\left[\left(\varepsilon_{1}+\varepsilon_{2}\right)\left(\frac{\omega_{c}}{\omega}\right)\right]-\aleph_{2}\left[\varepsilon_{1}+\varepsilon_{2}\left(\frac{\omega_{c}}{\omega}\right)^{2}\right]+\frac{\Delta\left(\omega^{2}-\omega_{h}^{2}\right)}{\omega_{p}^{2}}\left[k_{y}\left(\frac{\omega_{c}}{\omega}\right)-\aleph_{2}\right]\right\} \\
& +\frac{Z_{3}}{\chi_{2}}\left\{\left[\chi_{1}+\frac{\hbar}{m c}(\tanh \alpha)\right]\left[k_{y}\left(\frac{\omega_{c}}{\omega}\right)+\aleph_{v}\right]-\varepsilon_{2}\left[k_{y}+\aleph_{v}\left(\frac{\omega_{c}}{\omega}\right)\right]\right\}=0
\end{aligned}
$$

where,

$$
\begin{aligned}
& Z_{1}=\frac{\aleph_{2}+\aleph_{v}}{\aleph_{1}+\aleph_{v}}\left[\frac{\aleph_{2}^{2}-\aleph_{1}^{2}}{\aleph_{1}^{2}-k_{y}^{2}}-\left(\frac{\omega_{c}}{\omega}\right)\left(\frac{\chi_{3}}{\chi_{2}}\right) S\right], \\
& Z_{2}=\left(\frac{\aleph_{2}^{2}-k_{y}^{2}}{\aleph_{1}^{2}}\right)\left(\frac{\omega_{c}}{\omega}\right)\left(\frac{\chi_{3}}{\chi_{2}}\right) S, Z_{3}=\left(\frac{\omega_{c}}{\omega}\right)\left[1-\frac{\aleph_{2}+\aleph_{v}}{\aleph_{1}+\aleph_{v}}\right]
\end{aligned}
$$

The quantum corrections appear due to the spin effects, which are proportional to $\mu_{\beta}$ in the definition of $\tanh \alpha$, and due to the Bohm potential effect through the correction of $\hbar^{2}$ order in the definition of $Q$. When there is no background magnetic field, the effect of spin vanishes, but the quantum correction due to the Bohm potential will not vanish because it is independent to the coupling to the magnetic field. On the other hand, classical results are recovered when $\hbar \rightarrow 0$, obtaining the well-known dispersion relation for classical surface modes [28]:

$$
1-\frac{\omega_{p}^{2}}{\omega^{2}-\omega_{c}^{2}}-\frac{c^{2} k_{y}^{2}}{\omega^{2}}=0
$$

\section{Discussions}

The classical and the quantum corrected modes in magnetized or unmagnetized plasma are studied numerically through the dispersion relation (21) in this section. By taking the same procedure in Ref. [27], we are going to investigate the above dispersion relation by introducing the following normalized quantities $W=\omega / \omega_{p}$, $\bar{\omega}=\omega_{c} / \omega_{p}, K=c k / \omega_{p}, V=v_{F e} / c$ and $H=\hbar \omega_{p} / 2 m v_{F e}^{2}$ which describes the ratio of plasmonic energy density to the electron Fermi energy density. The values of magnetic field and number density are chosen for some magnetized dense astrophysical plasma.

In the first, we plot the dispersion relation (21) of the surface modes in Figure 1, for the simple case of unmagnetized plasma ( $\left.\omega_{c}=0\right)$, under different quantum effects $(H=1,2,3,4)$ and compare its behavior with the classical dispersion relation (Equation (22)) which is indicated by $(H=0)$ in this figure.

The most remarkable effects of quantum contribution is the increase in the effective group velocity ( $\partial \omega / \partial k$ ) and phase velocity of these modes which is due to the term of the Bohm potential. The implications of this quantum behavior in possible anomalous dispersion regions are under study. Besides, this effect in the phase and group velocities can lead to very exciting implications. 


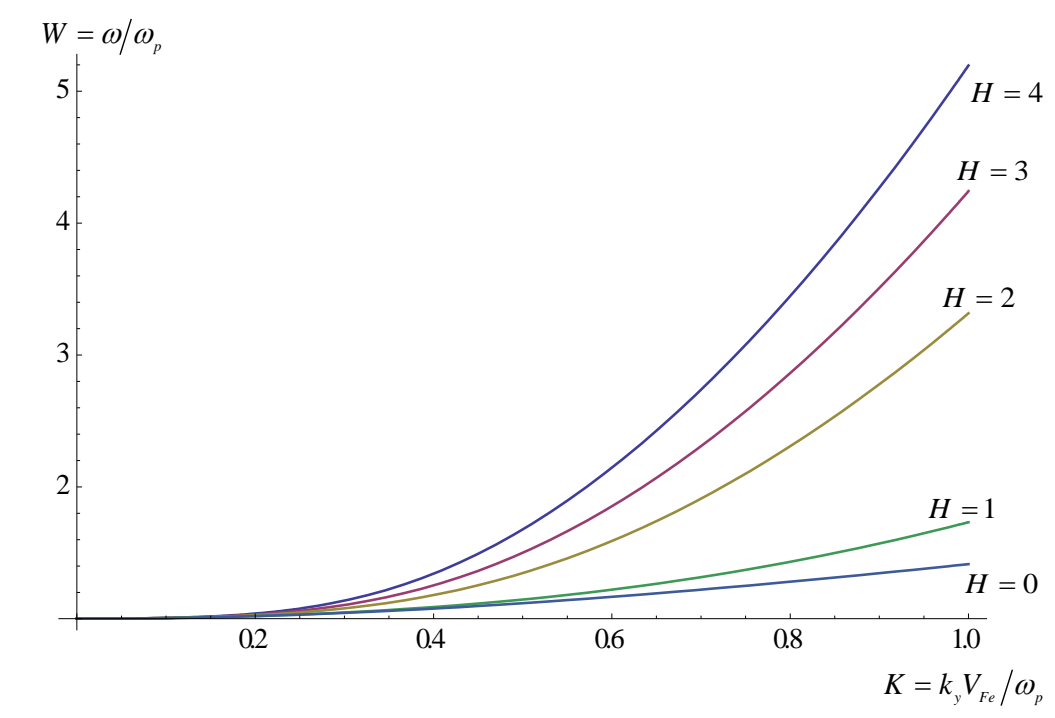

Figure 1. Dispersion relations of electromagnetic TM surface modes in quantum unmagnetized plasma ( $\bar{\omega}=0$ ) for different quantum effect ratios ( $H=0,1,2,3,4)$.

But in the case of quantum magnetized plasma, there are more different surface modes can be excited. So, we have numerically solved the dispersion relation (21) and some of these solutions are investigated in Figures 2(a)-(d). In the case of unmagnetized, the surface modes are initially excited with high frequency $\omega=\omega_{p}$ and are increased with increasing wavenumber. But, in the case of quantum plasma under external magnetic field, the surface modes (Figure 2) are initially excited with frequencies less plasma frequency $\omega<\omega_{p}$.

Figure 3 displays the dispersion relations of the excited surface modes in quantum magnetized spin plasma with different quantum ratios ( $H=1,2,3,4)$ for two solutions of Equation (21) (which are displaced in Figure 2(a) and Figure 2(b). The phase velocity of surface modes in Figure 3(a) is very high in the beginning of excitation and is decreased tends to zero with increasing the wavenumber. On the other side, the phase velocity of the modes in Figure 3(b) starts from zero in the beginning and increases with increasing wavenumber. But for all modes in two Figure 3(a) and Figure 3(b), it is clear that the phase velocity of these modes is monotonically increased with quantum ratio.

Besides, the effect of external magnetic field on the excitation of TM surface waves is investigated in the Figure 4. The magnetic field makes increasing the frequency of these modes in the beginning of excitation. It is also noted that the phase velocity of the modes tends to zero along the wavenumber for all values of external magnetic field ( $\bar{\omega}=0.1,0.2,0.3$ ).

A comparison between the dispersion of the modes in quantum magnetized plasma with and without spin effects is investigated in Figure 5. The solution of the dispersion displaced in the Figure 2(d) is studied and compared with the solution of the relation (21) without spin effects (by assuming $\tanh \alpha=0$ ). It has been shown that the influence of electron spin produces an increasing in the phase velocity and effective group velocity of the surface modes for small wave number, but this increasing is not enough to produce an anomalous dispersion effect. It is also noticed that the frequency of the surface mode initially excited in the spin case is bigger than the frequency in the case of without spin effect due to the addition of magnetization term of spin.

\section{Conclusion}

To summarize, we have solved the full set of equations of quantum hydrodynamic model which describes the spin dynamics of electrons. The quantum corrections to the excitation and propagation of surface waves in quantum plasma under an external magnetic field have been investigated. These corrections are due to Bohm potential and to spin of electrons. It showed how contribution of both quantum effects modifies the plasma current density and thereby introduces a correction term in the dispersion relation, which, in turn gives rise to new modes. The dispersion relation for these waves is solved numerically for normal variables of frequency and wave number. This dispersion equation is studied for different cases and investigated graphically (classical or 


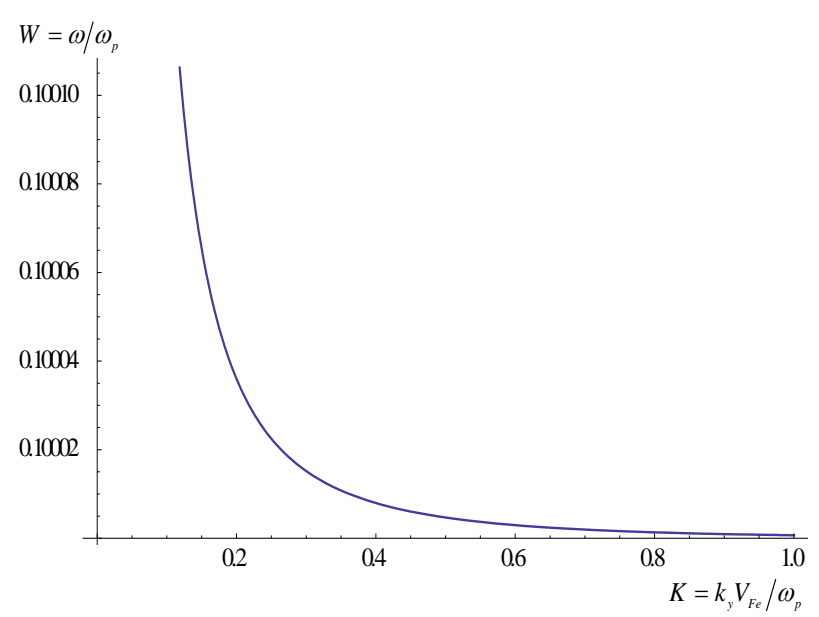

(a)

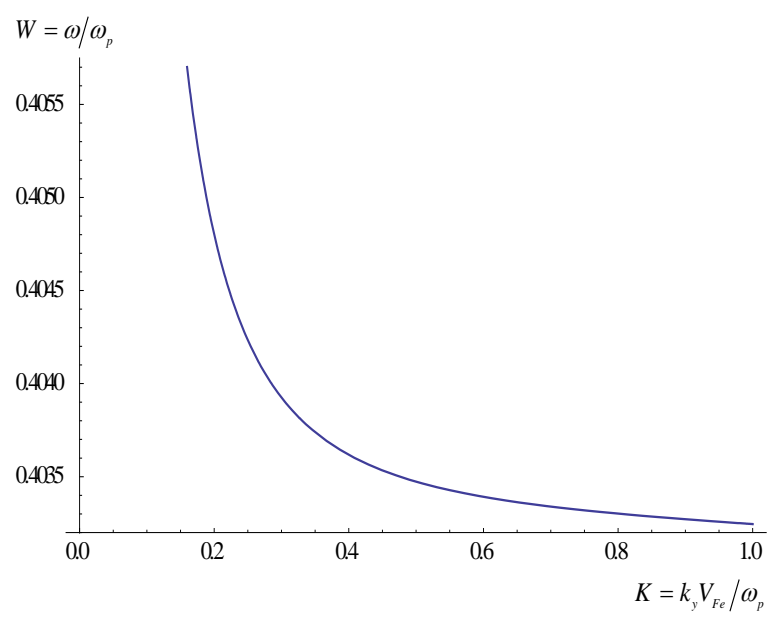

(c)

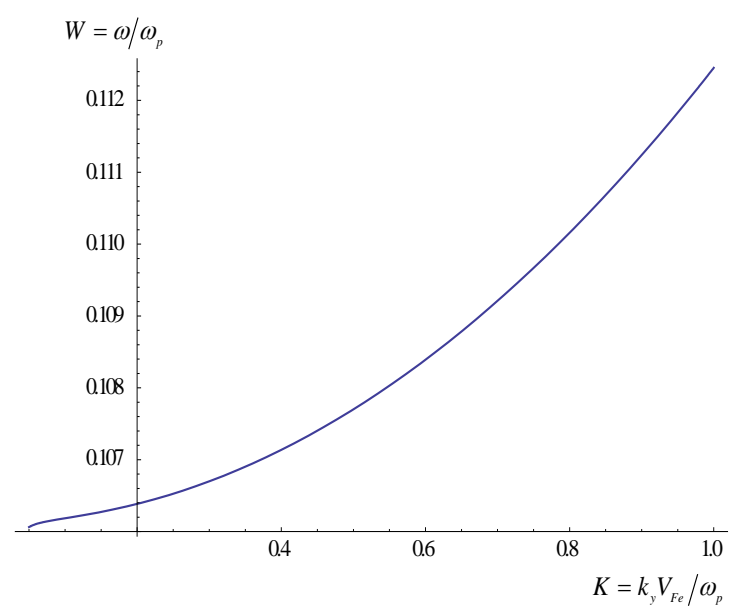

(b)

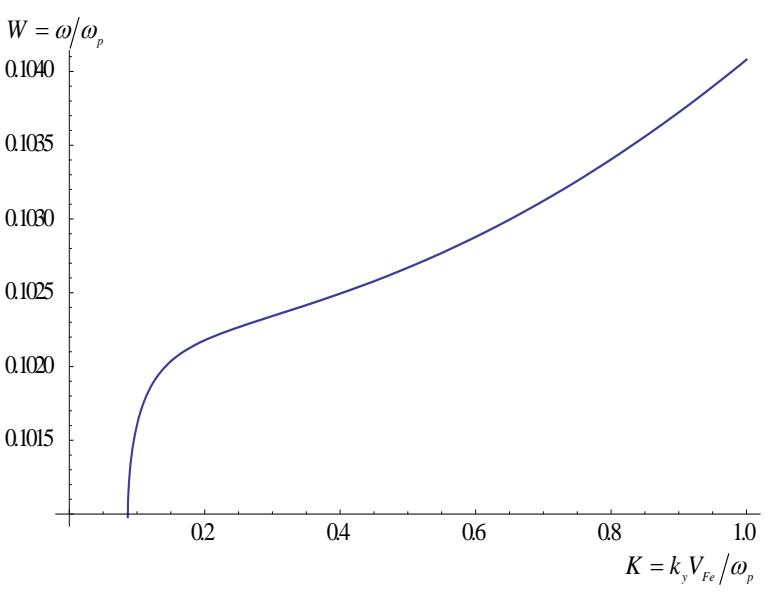

(d)

Figure 2. Different solutions ((a), (b), (c) and (d)) for dispersion relation (21) of electromagnetic surface modes in quantum magnetized plasma.

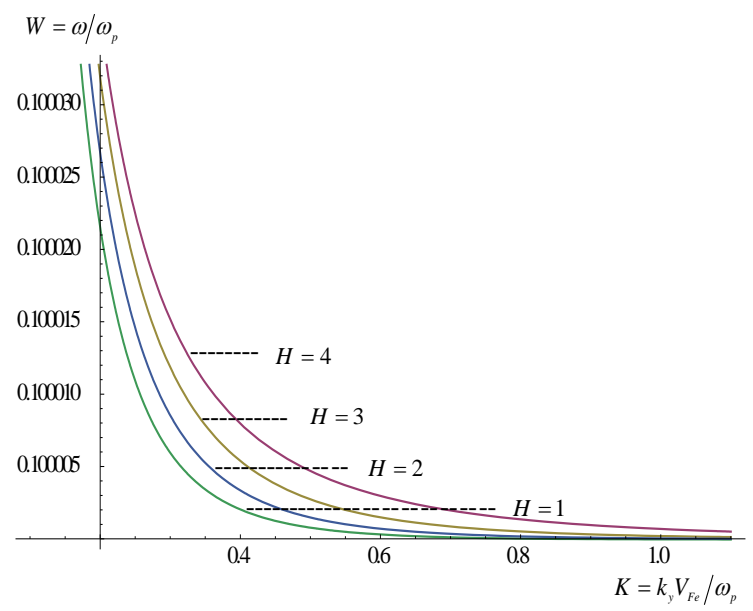

(a)

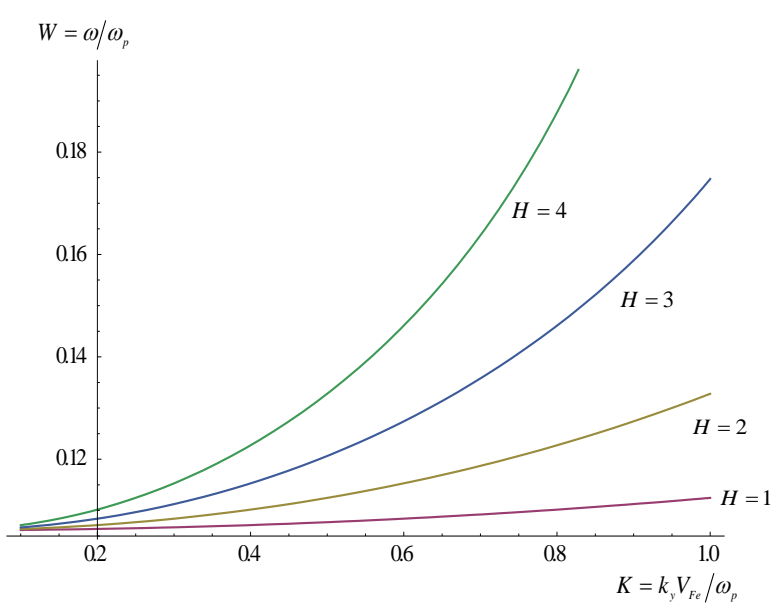

(b)

Figure 3. Two different solutions of dispersion relation (21) for electromagnetic TM surface modes in quantum magnetized plasma ( $\bar{\omega}=0.1$ ) for different quantum ratios $(H=1,2,3,4)$. 


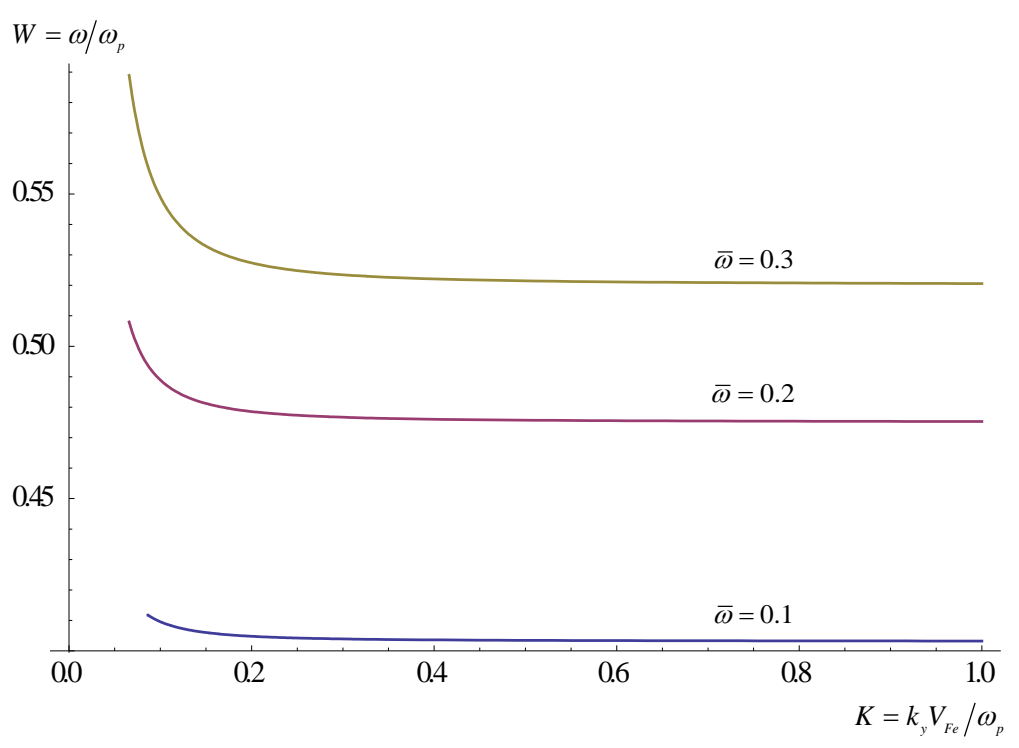

Figure 4. Dispersion relations of electromagnetic TM surface modes in quantum magnetized plasma ( $\bar{\omega}=0.1,0.2,0.3)$ with quantum ratio $(H=1)$.

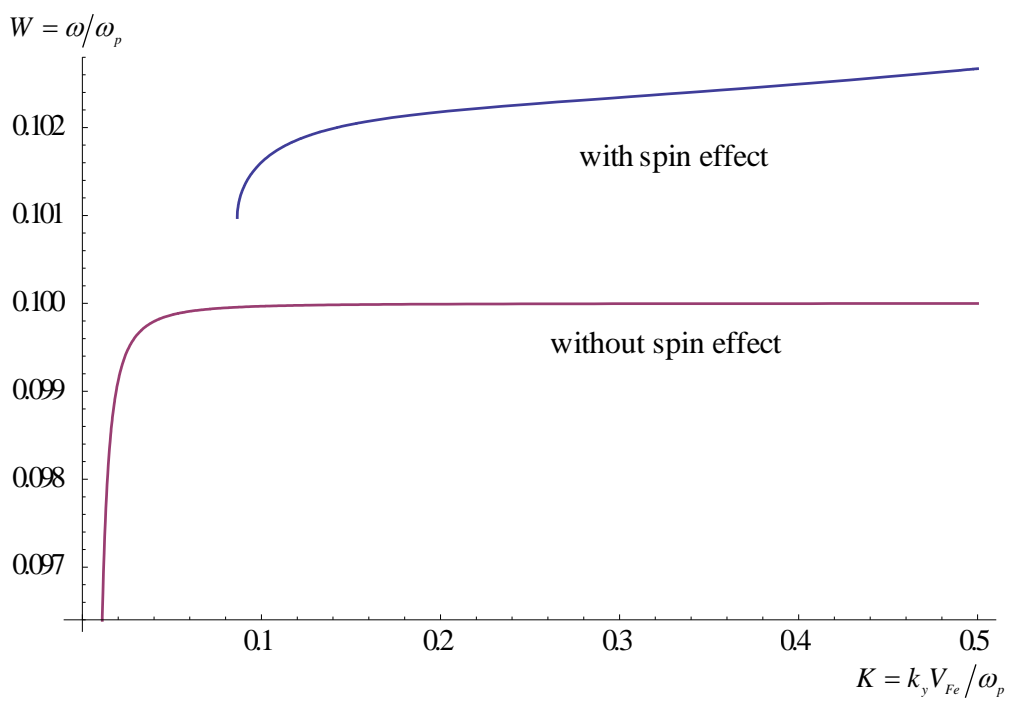

Figure 5. Solution of dispersion relation (21) with and without spin effect of electromagnetic surface modes in quantum magnetized plasma ( $\bar{\omega}=0.1$ and $H=1$ ).

quantum, magnetized or unmagnetized and with or without spin effect). It is found that the phase and group velocities are increased due to the quantum effects. It is also noted that increase of magnetic field tends to increase the frequency of the excited modes. Besides, when the spin effect is taken into account, it is similar to the effect of increasing magnetic field.

\section{References}

[1] Brodin, G. and Marklund, M. (2007) Physical Review E, 76, Article ID: 055403. http://dx.doi.org/10.1103/PhysRevE.76.055403

[2] Shukla, P. and Eliasson, B. (2011) Reviews of Modern Physics, 83, 885. http://dx.doi.org/10.1103/RevModPhys.83.885

[3] Brodin, G. and Marklund, M. (2007) Physics of Plasmas, 14, Article ID: 112107. http://dx.doi.org/10.1063/1.2793744

[4] Misra, A. and Samanta, S. (2010) Physical Review E, 82, Article ID: 037401. 
[5] Brodin, G., et al. (2011) Plasma Physics and Controlled Fusion, 53, Article ID: 074013. http://dx.doi.org/10.1088/0741-3335/53/7/074013

[6] Asenjo, F.A., et al. (2011) Physics of Plasmas, 18, Article ID: 012107. http://dx.doi.org/10.1063/1.3533448

[7] Mahajan, S.M. and Asenjo, F.A. (2013) Physics Letters A, 377, 1430-1433. http://dx.doi.org/10.1016/j.physleta.2013.04.021

[8] Krishnaswami, G.S., et al. (2013) arXiv:1306.1774.

[9] Shukla, P.K. and Eliasson, B. (2010) Physics-Uspekhi, 53, 51. http://dx.doi.org/10.3367/UFNe.0180.201001b.0055

[10] Asenjo, F.A., et al. (2012) New Journal of Physics, 14, Article ID: 073042. http://dx.doi.org/10.1088/1367-2630/14/7/073042

[11] Kharzeev, D.E., Landsteiner, K., Schmitt, A. and Yee, H.-U. (2013) Strongly Interacting Matter in Magnetic Fields: A Guide to This Volume. Springer, Berlin. http://dx.doi.org/10.1007/978-3-642-37305-3

[12] Shukla, P. (2007) Physics Letters A, 369, 312-314. http://dx.doi.org/10.1016/j.physleta.2007.04.091

[13] Misra, A.P., Brodin, G., Marklund, M. and Shukla, P.K. (2010) Physics of Plasmas, 17, Article ID: 122306. http://dx.doi.org/10.1063/1.3527995

[14] Rabl, P., Kolkowitz, S.J., Koppens, F.H.L. et al. (2010) Nature Physics, 6, 602-608. http://dx.doi.org/10.1038/nphys1679

[15] Brodin, G. and Marklund, M. (2007) New Journal of Physics, 9, 277. http://dx.doi.org/10.1088/1367-2630/9/8/277

[16] Sachdev, S. and Keimer, B. (2011) Physics Today, 64, 29. http://dx.doi.org/10.1063/1.3554314

[17] Prajapati, R. and Chhajlani, R. (2010) Physica Scripta, 82, Article ID: 055003. http://dx.doi.org/10.1088/0031-8949/82/05/055003

[18] Keane, A., Mushtaq, A. and Wheatland, M. (2011) Physical Review E, 83, Article ID: 066407. http://dx.doi.org/10.1103/PhysRevE.83.066407

[19] Braun, S., Asenjo, F.A. and Mahajan, S.M. (2012) Physical Review Letters, 109, Article ID: 175003. http://dx.doi.org/10.1103/PhysRevLett.109.175003

[20] Shukla, P.K. and Eliasson, B. (2012) Physical Review Letters, 108, Article ID: 165007. http://dx.doi.org/10.1103/PhysRevLett.108.165007

[21] Mohamed, B.F. and Abdel Aziz, M. (2010) International Journal of Plasma Science and Engineering, 2010, Article ID: 693049, 4 p.

[22] Lundin, J. and Brodin, G. (2010) Physical Review E, 82, Article ID: 056407. http://dx.doi.org/10.1103/PhysRevE.82.056407

[23] Brodin, G., Marklund, M., Zamanian, J., et al. (2008) Physical Review Letters, 101, Article ID: 245002. http://dx.doi.org/10.1103/PhysRevLett.101.245002

[24] Brodin, G., Misra, A.P. and Marklund, M. (2010) Physical Review Letters, 105, Article ID: 105004. http://dx.doi.org/10.1103/PhysRevLett.105.105004

[25] Misra, A.P., Brodin, G., Marklund, M. and Shukla, P.K. (2010) Physical Review E, 82, Article ID: 056406. http://dx.doi.org/10.1103/PhysRevE.82.056406

[26] Asenjo, F.A. (2012) Physics Letters A, 376, 2496-2500. http://dx.doi.org/10.1016/j.physleta.2012.06.023

[27] Mohamed, B.F. and Elbasha, N.M. (2015) Physics of Plasmas, 22, Article ID: 102101. http://dx.doi.org/10.1063/1.4931995

[28] Mohamed, B.F. (2010) Physica Scripta, 82, Article ID: 065502. http://dx.doi.org/10.1088/0031-8949/82/06/065502 